\title{
Identification of Single Adsorbed Cations on Mica- Liquid Interfaces by 3D Force Microscopy
}

Daniel Martin-Jimenez, Ricardo Garcia*

Materials Science Factory, Instituto de Ciencia de Materiales de Madrid, CSIC, c/ Sor Juana Ines de la Cruz 3, 28049 Madrid, Spain

r.garcia@csic.es 


\section{ABSTRACT}

Force microscope provides atomically-resolved images of surfaces immersed in a liquid. The presence of different chemical species in the interface (cations, anions, water, neutral atoms) complicates the adscription of the observed features to a given specie. Here, we develop a threedimensional atomic force microscopy method to identify the cations adsorbed on a mica surface from a potassium chloride solution. The method is based on measuring the peak value of the attractive force within the Stern layer. The maximum of the attractive force shows site-specific variations. The positions with the highest attractive force values are associated with the presence of adsorbed potassium ions while the other positions are associated with a local depletion of the hydration layer. This criterion provides a surface coverage of $\mathrm{K}$ cations that is consistent with the one reported by other techniques.

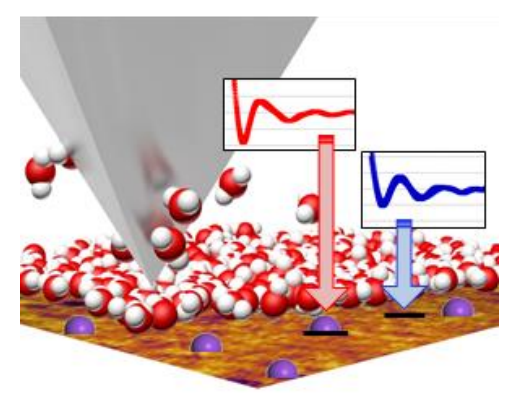


Dynamic atomic force microscopy (AFM) methods are used to study different processes happening at solid-liquid interfaces. AFM has provided atomically-resolved images of diffusion $^{1}$, crystal growth ${ }^{2}$ or etching ${ }^{3}$. In an aqueous solution, the interactions among the surface atoms, the ions from the solution and the water molecules could lead to the structuring of the interface near the solid surface $e^{4-12}$.

Very close to the solid surface, say below $1 \mathrm{~nm}$, some of the dynamic AFM observables, notably, the frequency shift ${ }^{13}$, amplitude ${ }^{14}$ or the phase shift ${ }^{15}$ show some oscillations as the separation is decreased. Those observables enable to determine the dependence of the force with respect to the separation of the solid surface (force-distance curve). For aqueous solutions, the oscillations in force-distance curves are attributed to changes in the water density, although its detailed structure also reflects the type and charge of ions that are present in the interface ${ }^{16-}$ 22.

It has been proposed that the site-specific differences observed in the force-distance curves could be used for the chemical identification of the different species on the surface ${ }^{17,19,20}$. However, this approach might require the use of molecular dynamics (MD) simulations. In addition, on equivalent lattice positions, the amplitude and positions of the peaks and valleys of the force-distance curves might change. Those changes could be caused by either instrumental noise or tip induced fluctuations of the liquid near the solid surface. Topographic images have also been used to identify adsorbed cations ${ }^{1,23}$. However, the use of topography to identify a given chemical species has limitations. Different chemical specifies could give rise to similar topographic changes. In general, it is hard to identify the different chemical species 
present on a solid-liquid interface with the same spatial resolution provided by the AFM topography images (atomic).

A mica- $\mathrm{KCl}$ (aqueous) solution interface provides a paradigmatic example of some of the above issues. Atomic-resolution images of the mica surface in the presence of different solutions have been reported by different AFM methods such as contact mode ${ }^{24}$, frequency modulation ${ }^{25}$, amplitude modulation $^{26}$ or bimodal ${ }^{27}$. Most AFM images report a hexagonal lattice although, in some cases, honey-comb patterns are observed. It has also been observed the transition between an hexagonal lattice to a honey-comb pattern by changing the operation parameters (set-point amplitudes or frequency shifts). In the presence of a $\mathrm{KCl}$ solution, the atomic-like features of the hexagonal pattern observed in AFM images are often attributed to the cations adsorbed from the alkali halide solution. However, this interpretation is at odds with X-ray reflectivity data that, for similar concentrations, shows the mica surface covered by a half monolayer of monovalent cations ${ }^{28-30}$.

Three-dimensional AFM (3D AFM) is a method to characterize the structure of a solid-liquid interface in the three spatial coordinates ${ }^{16}$. This method provides atomically-resolved images of solid-liquid interfaces on different crystalline surfaces such as mica ${ }^{15,16,31,32}$, calcite ${ }^{17,20}$, fluorite $^{18,22}$ or sodium chloride ${ }^{21}$. It has also been applied to image soft matter-water interfaces on purple membrane ${ }^{32}$, proteins $^{15}$ or lipid bilayers ${ }^{33}$. The depth of the $3 \mathrm{D}$ images has been increased from less than $1 \mathrm{~nm}$ reported in the first 3D AFM cross-section ${ }^{16}$ to above $10 \mathrm{~nm}$ obtained by operating the instrument in the amplitude modulation mode ${ }^{34}$. Some 3D AFM 
configurations enable to record the force-distance curve $\mathrm{e}^{15,16,31,34}$ at the same time that the $3 \mathrm{D}$ map is recorded.

Here we demonstrate the capability of 3D AFM to identify the cations species adsorbed within the Stern layer. The method measures and classifies the peak attractive force values on each position of the layer. On equivalent positions of a mica surface immersed on a $\mathrm{KCl}$ solution, we found that the peak value could change by a factor 2 from one position to the next. The atomic positions with the highest peak attractive force values are associated with the presence of adsorbed potassium ions while the other positions are associated with a local depletion of the water density.

The cleaved surface of muscovite mica is formed by an aluminosilicate layer arranged in a honeycomb structure (Figure 1a). One out of four Si atoms is replaced by an Al atom. The presence of $\mathrm{Al}$ generates a negative surface charge density of one electron per unit cell. Within the crystal, the negative charge is cancelled by the layers including $\mathrm{K}$ cations. Once the mica is cleaved, half of the mica cavities remain filled with $\mathrm{K}$ ions. This assures the electrical neutrality of the surface (Figure 1a). If the surface is immersed in an aqueous solution, some $\mathrm{K}$ ions could diffusive away from the surface. In $\mathrm{KCl}$ aqueous solutions, the surface charge will be neutralized by the adsorption of $\mathrm{K}$ ions within the Stern layer (Figure 1b). At very low molarities, say below $1 \mathrm{mM}$, a different process could shield the negatively charged mica surface. Water cations either located in the Stern layer or within the diffusive layer ${ }^{5,28-30}$ could shield the charge (Figure 1b). Figure 1c and d show, respectively, the mica surface and the interface (cross-section) with the different atoms plotted with their van der Waals radius.

The 3D AFM is operated with a loose amplitude modulation feedback AFM. In each voxel of the interface, the amplitude $(A)$ and phase shift $(\phi)$ of the tip's oscillation are recorded. An 
amplitude modulation feedback provides a robust 3D AFM operation. This approach enables to acquire images relatively far from the mica surface ${ }^{34}$ at the same time that force-distance curves are recorded (see Methods).

Figure 2a shows a 3D image of a mica-KCl solution interface for a $200 \mathrm{mM}$ concentration. The outermost mica surface is shown in yellow while the $\mathrm{KCl}$ (aqueous solution) interface appears in blue (color-coded phase shift values). The position of the mica surface plane is readily identified because by approaching the tip towards the surface, the force becomes increasingly repulsive. Above the mica surface, the 3D phase shift map shows a layered structure that alternates dark and blue regions. This structure is observed within $1 \mathrm{~nm}$ from the mica surface. Figures 2b-c display two $x z$ planes at different $y$ positions (marked in Figure 2a). The $x z$ maps show the alternation of layers of about $0.3 \mathrm{~nm}$ in width with different phase shift values. The theory of phase imaging $\mathrm{AFM}^{35,36}$ establishes that an attractive force produces a phase shift value above $90^{\circ}$ (dark blue). Regions of the interface characterized by a net negative force produce a phase shift below $90^{\circ}$ (light blue). Consequently, those layers are associated with either attractive or repulsive forces. In addition, we observe that the layer closer to the mica surface is discontinuous. It shows the presence of site-specific atomic positions with different phase shift contrast. Some of those positions are marked by circles in Figures $2 \mathrm{~b}$ and $\mathrm{c}$.

The 3D phase shift map (Figure 2a) can be transformed into quantitative force-distance curves by processing the changes of the amplitude and phase shift along the $z$-axis ${ }^{37,38}$. Figures $2 \mathrm{~d}$ and 2e show the force-distance curves obtained at some specific positions of the interface ( 1 and 2 in panel $2 \mathrm{~b}$ and 3 and 4 in panel $2 \mathrm{c}$ ). The force curves alternate attractive and repulsive regions. Far from the mica surface $(z \geq 1 \mathrm{~nm})$, the force is within the noise level $(0 \pm 20 \mathrm{pN})$. Between 
0.7-0.8 $\mathrm{nm}$ a small peak in the repulsive force is observed. This is followed by a region of attractive forces centered at $z=0.60-0.65 \mathrm{~nm}$. The force becomes repulsive again with a maximum at $z=0.45 \mathrm{~nm}$. By keeping the tip moving towards the mica surface, the force becomes attractive until it reaches a maximum (absolute value) at $z=0.3 \mathrm{~nm}$. From there on, the force rapidly becomes repulsive. The monotonic increase of the repulsive force indicates the position of the mica surface. The four curves marked in Figures $2 b$ and $2 c$ show similar qualitative features. However, there are also some remarkable quantitative differences. First, the values of the local maxima and minima depend on curve. Second, the curves can be classified in two groups according to the value of the maximum attractive force. Those forces are centered, respectively, at -370 (Figure $2 \mathrm{~d}$ ) and $-200 \mathrm{pN}$ (Figure 2e). A more complete statistical analysis is provided below.

The interpretation of the features (attractive and repulsive peaks) of the above force-distance curves is under a theoretical scrutiny ${ }^{17,18}$. It can be said that the repulsive peaks are produced by an increase in the confinement of the water distribution under the tip. The attractive peaks (other than the one near the mica surface) are produced by a re-organization of the hydration structure. Some simulations performed for a calcite-water interface have underlined the similarity between the water density and the force-distance curves. However, on other interfaces such as fluorite-water, the force peaks follow the peaks on the logarithmic derivative of the density with respect to tip-sample distance ${ }^{18}$. In short, the theoretical descriptions enable to label the regions reported here. The strip closer to the mica surface corresponds with the transition between the first hydration layer and the mica surface. Its discontinuous character indicates that this region includes positions where the presence of water molecules is unlikely. The next layer 
corresponds to the transition between the first and second hydration layer while the dark band corresponds to a transition from the depletion layer to the second hydration layer. The above interpretation provides qualitative description of the observed features; however, it does not explain the discontinuities observed of within the first layer. It fails to explain the origin of the differences observed in the values of the peak attractive force (see Figures $2 \mathrm{~d}$ and e). Furthermore, it does not assign a given feature to an adsorbed cation.

In fact, the differences observed in the peak value of the attractive force allow us to propose a method to distinguish between the different atomic positions of the solid-liquid interface. Figure 3a shows a 2D peak attractive force map. The 2D peak force map corresponds to a $x y$ section of the 3D map taken within the Stern layer (this 2D map includes the horizontal lines marked in Figures $2 \mathrm{~b}$ and c). By overlaying the lattice structure of mica (Figure 1a without K cations), we observe that the peak force values are included within the mica cavities. Figures $3 \mathrm{~b}$ and $\mathrm{c}$ shows the force-distance curves taken on the mica cavities. The force curves have been classified in two classes according the peak value of the attractive force. One is centered around $-355 \mathrm{pN}$ (Figure 3b) and the other at $-192 \mathrm{pN}$ (Figure 3c). We observe that the spatial width of the local minima or maxima in those curves ( $\Delta z$ error in the position of a given peak) increases with the distance from the mica surface. For the first minima $(z=0.25 \mathrm{~nm}) \Delta z=0.04 \mathrm{~nm}$, for the maximum at $z=0.38 \mathrm{~nm}$ we get $\Delta z=0.08 \mathrm{~nm}$, while for last oscillations the error is $\Delta z=0.12$ nm.

From the above observations we propose a method to label the atomic-like features observed in the Stern layer (Figure 3a). The method measures the peak value of the attractive force as given from a force-distance curve. Then, Figure 3 a could be interpreted in terms of the location of the 
adsorbed cations $\left(\mathrm{K}^{+}\right)$. The positions that show a peak value centered at $-355 \pm 40 \mathrm{pN}$ (red) are associated with the presence of an adsorbed cation. On the other hand, the points in the image that have peak values centered at $-192 \pm 40 \mathrm{pN}$ (blue) are associated with a depletion of the water density. The above peak values are specific of the local tip geometry used in the experiment.

We emphasize that peak attractive force value provides a robust method to identify the cation. The $z$-dispersion in the position of this peak is smaller than the ones of the other peaks. Also, the peak value of the attractive force is less sensitive to changes in the interface due to either the diffusion or desorption of other cations or water molecules. We also remark that the interaction of the tip with the adsorbed ions on the mica is a dynamical process. There are examples of force-distance curves where an adsorbed cation could be removed by the interaction with the tip (See Figure S2).

The peak observed in the attractive force near the mica surface can be explained in terms of the electrostatic interaction between the tip and a given site of the interface. It has been established that silicon tips are negatively charged in our experimental conditions $(\mathrm{pH}=5.5)^{7,8}$. Within the Stern layer, when the tip is directly on top of a cation the electrostatic attractive is maximized with respect to the other atomic sites. This interpretation is consistent with the surface coverage obtained by other means. In the presence of a $200 \mathrm{mM}$ solution of $\mathrm{KCl}$ (aqueous), the 2D peak force maps show that about half of the mica surface is covered with positions with highest peak attractive force values. This result is agreement with the cation coverage (0.5 ML) determined by X-ray diffraction ${ }^{28-30}$. It coincides with the nominal surface charge density needed to shield the negative charge of the mica surface after cleavage. Our interpretation shows that the cations form geometrical domains (Fig. 3c). This observation is also in agreement with other studies involving $\mathrm{Rb}$ cations on mica $^{5}$. 
Our interpretation is also consistent with some MD simulations of solid-liquid interfaces that consider the chemical and geometrical properties of the AFM tip. The forces acting between ionic surfaces and either positive or negatively charged tips have been calculated ${ }^{17-19,40,41}$. The simulations show that outside the Stern layer, the contribution to the total force arising from electrostatic interactions could be neglected. However, the electrostatic interactions become dominant when the separation between the tip and an ion is below the diameter of a water molecule. In this case, there is a direct interaction between the tip's and the adsorbed ions or atoms ${ }^{18,40}$.

In short, we present a method to identify the positions of individual cations within the Stern layer above a mica surface. Three-dimensional AFM images of the solid-liquid interface enable the determination of the force-distance curve on each point of the surface. We observe a peak in the attractive force before the tip interacts with the mica surface. This peak shows sitespecific values. The positions with the highest attractive force values are associated with the presence of adsorbed potassium ions while the other positions are associated with local depletions of the hydration layer. The method could be extended to other type of cations and surfaces.

\section{EXPERIMENTAL METHODS}

Three-dimensional AFM operation was implemented on a Cypher AFM ( Asylum Research, Oxford Instruments) by means of a home-made software to control the electronics and the tip motion of the AFM. The oscillation of the cantilever was driven by photothermal excitation at a wavelength of $405 \mathrm{~nm}$. 
Three-dimensional images of mica-liquid interface are performed by operating the AFM in the amplitude modulation mode. While the tip oscillates, a sinusoidal signal is applied to change the $z$ position of the tip during the acquisition of a $x y z$ image. The $z$ motion of the tip is synchronized with the $x y$ trajectory.

The amplitude modulation feedback tracks the instantaneous amplitude to keep it at a fixed value. The feedback bandwidth is relatively small $\sim 1 \mathrm{kHz}$. This means a rather slow feedback response (loose feedback). The $z$ signal has a period of $20.5 \mathrm{~ms}$. The $z$ data is stored in 1024 pixels (512 pixels half cycle). Each $x y$ plane of the 3D map contains $80 x 64$ pixels. The total time to acquire a 3D AFM map is of $105 \mathrm{~s}$.

The force-distance curves are determined by processing the dependence of the amplitude and phase shift observables with the tip-sample separation. This requires the use of force reconstruction methods ${ }^{37,38}$. Here we have used Holscher's method ${ }^{37}$.

Discs of muscovite mica (Grade V-1, SPI supplies, USA) were cleaved with adhesive tape before the experiments. A solution of $200 \mathrm{mM} \mathrm{KCl}$ was prepared with $\mathrm{KCl}$ salt (Sigma Aldrich) dissolved in ultrapure water (18.2 $\mathrm{M} \Omega, \mathrm{pH}=5.5)$. A droplet of the solution was deposited on a freshly cleaved mica. The sample was placed in the microscope and the probe was immersed inside the solution.

Silicon cantilevers PPP_NCHAuD (Nanosensor, Germany) were used for 3D AFM imaging. The force constant, resonant frequency and quality constant of the second eigenmode were determined in the solution. Their values were $k_{2}=858 \mathrm{~N} / \mathrm{m}, f_{2}=811 \mathrm{KHz}, Q_{2}=21$. For imaging, the free and set-point amplitudes were in the sub-angstrom regime to be able to detect variations within the Stern layer, respectively, of $72 \mathrm{pm}$ and $65 \mathrm{pm}$. 

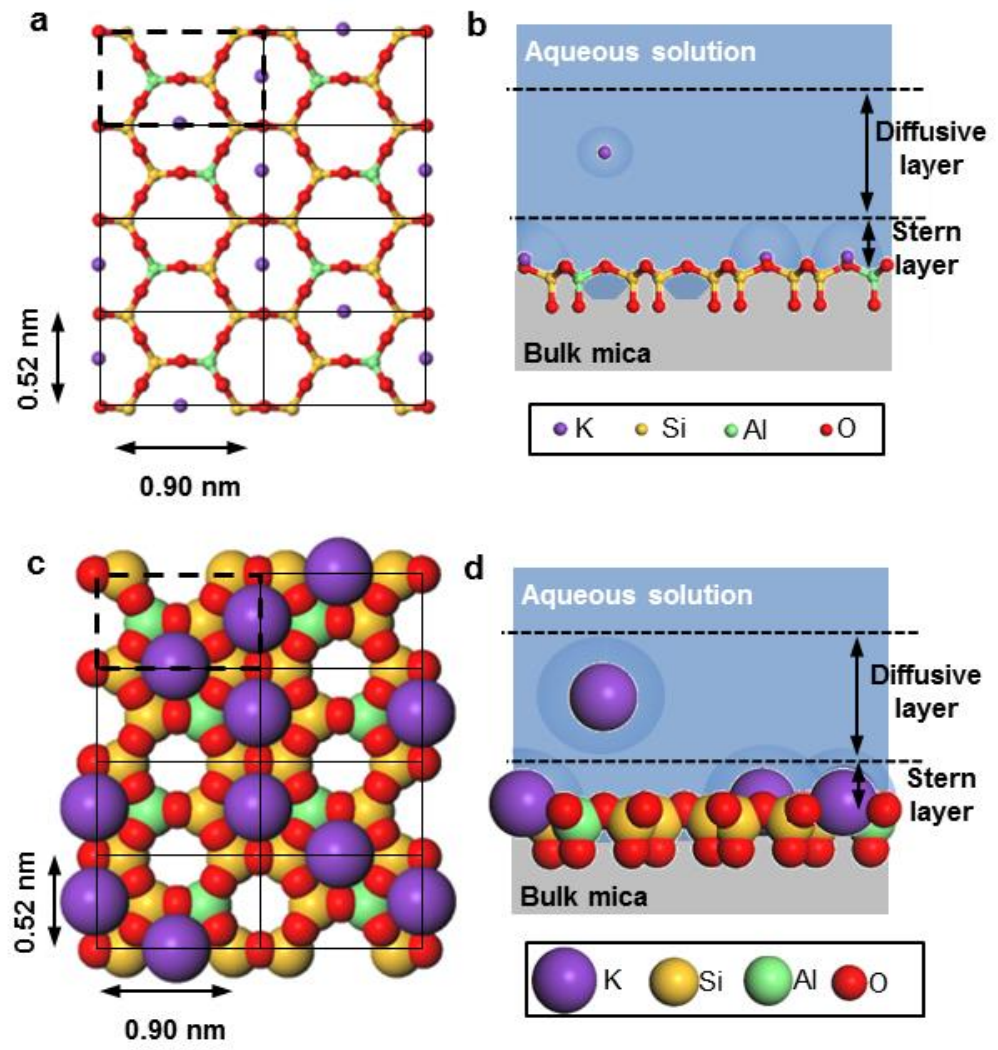

Figure 1. a. Scheme of the mica (001) surface after cleavage. b. Cross-section of a mica-aqueous solution interface. c. Same as panel $a$. The size of the atoms represents the van der Waals diameters. d. Same as $b$ with the atoms plotted at their respective van der Waals diameters. 

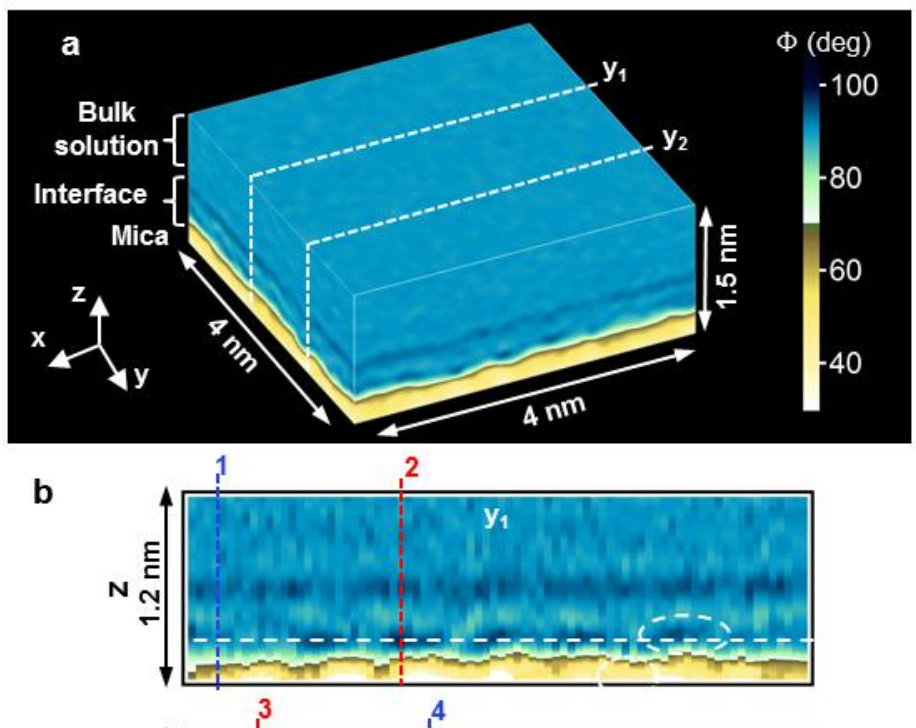

c
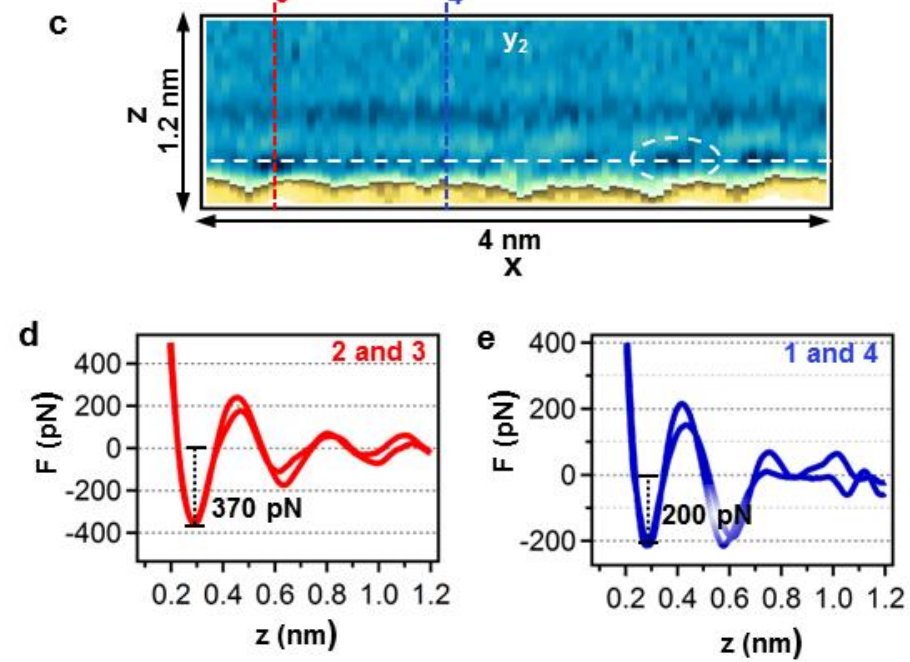

Figure 2. a Three-dimensional AFM image of the mica-liquid interface ( $200 \mathrm{mM} \mathrm{KCl})$. The map shows the changes of the phase shift of the tip's oscillation as it moves in a $x y z$ volume of the interface. The dashed lines indicate the $y$ positions at which two $x z$ maps were obtained. $\mathbf{b} x z$ map of the mica-KCl interface at $y_{1}$ in panel $a$. c $x z$ map of the mica- $\mathrm{KCl}$ interface at $y_{2}$ in panel $a$. d Force-distance curves taken along some of the lines marked in panels $b$ and $c$. e Force-distance curves taken along some of the lines marked in panels $b$ and $\mathrm{c}$. 

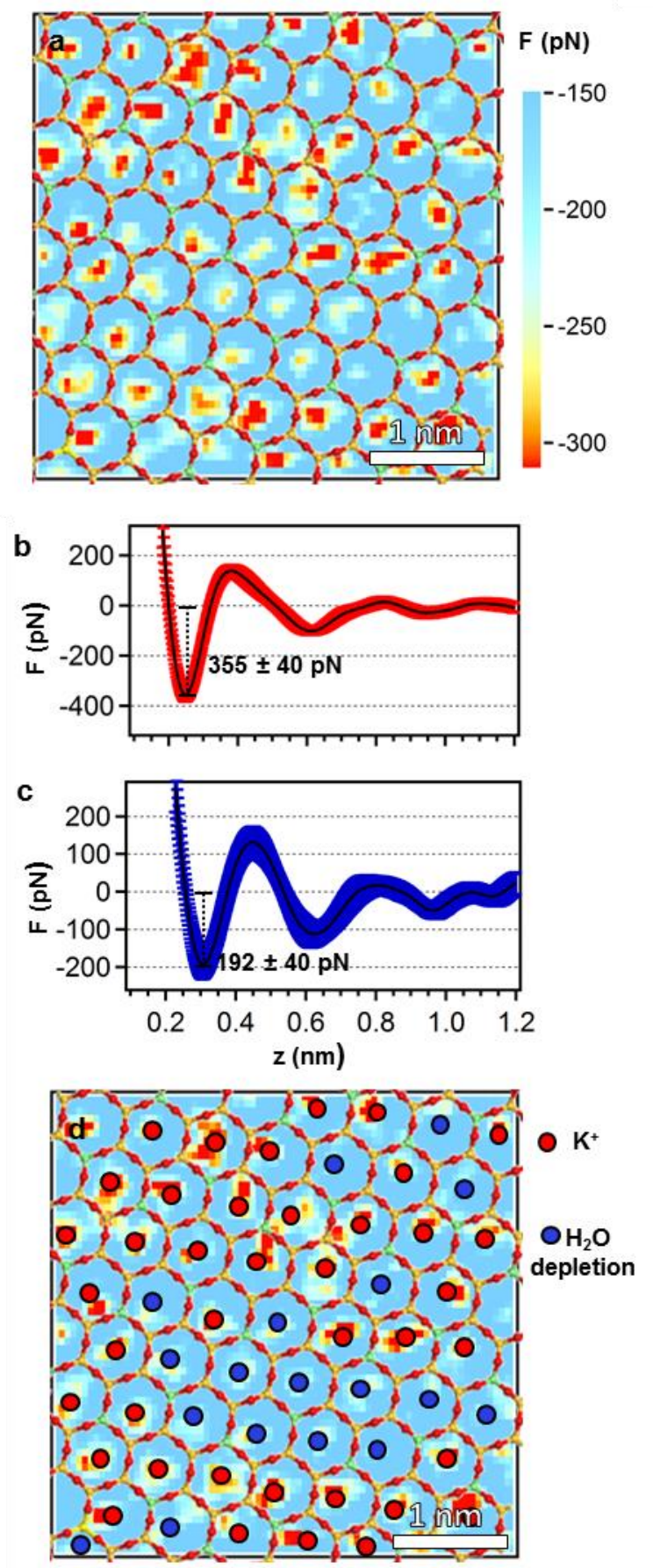

Figure 3. a $x y$ map at $z=0.3 \mathrm{~nm}$. It includes the horizontal dashed line marked in Figures $2 \mathrm{~b}, \mathrm{c}$. We have overlaid the mica (001) structure (without K cations). b Force-distance curves taken on the orange dots within the cavities of Figure 3a. c Force-distance curves taken on the yellow dots within the cavities of Figure 3a. The continuous line (black) shows the average value of the forcedistance curve. $x y$ map with the positions of the $\mathrm{K}$ cations marked in red. Those positions have the highest peak force values $(F \approx-355 \mathrm{pN})$. In blue we mark the regions that show a depletion of the water density with respect to the bulk $(F \approx-192 \mathrm{pN})$. 


\section{AUTHOR INFORMATION}

Corresponding author:

*E-mail: r.garcia@csic.es

ORCID

Ricardo Garcia: 0000-0002-7115-1928

\section{Notes}

The authors declare no competing financial interests.

\section{ACKNOWLEDGMENT}

This work was funded by the European Research Council ERC-AdG-340177 (3DNanoMech) and Spanish Ministry of Science and Innovation (MAT2016-76507-R). 


\section{REFERENCES}

(1) Ricci, M.; Trewby, W.; Cafolla, C.; Voïtchovsky, K. Direct Observation of the Dynamics of Single Metal Ions at the Interface with Solids in Aqueous Solutions. Sci. Rep. 2017, 7 , 43234.

(2) Miyata, K.; Tracey, J.; Miyazawa, K.; Haapasilta, V.; Spijker, P.; Kawagoe, Y.; Foster, A. S.; Tsukamoto, K.; Fukuma, T. Dissolution Processes at Step Edges of Calcite in Water Investigated by High-Speed Frequency Modulation Atomic Force Microscopy and Simulation. Nano Lett. 2017, 17, 4083-4089.

(3) Chiodini, S.; Reinares-Fisac, D.; Espinosa, F. M.; Gutiérrez-Puebla, E.; Monge, A.; Gándara, F.; Garcia, R. Angstrom-Resolved Metal-Organic Framework-Liquid Interfaces. Sci. Rep. 2017, 7, 11088.

(4) Araki, Y.; Satoh, H.; Okumura, M.; Onishi, H. Localization of Cesium on Montmorillonite Surface Investigated by Frequency Modulation Atomic Force Microscopy. Surf. Sci. 2017, 665, 32-36.

(5) Ricci, M.; Spijker, P.; Voïtchovsky, K. Water-Induced Correlation between Single Ions Imaged at the Solid-Liquid Interface. Nat. Commun. 2014, 5, 4400.

(6) Siretanu, I.; Ebeling, D.; Andersson, M. P.; Stipp, S. S.; Philipse, A.; Stuart, M. C.; Van den Enden, D.; Mugele, F. Direct Observation of Ionic Structure at Solid-Liquid Interfaces: a Deep Look into the Stern Layer. Sci. Rep. 2014, 4, 04956.

(7) Umeda, K.; Kobayashi, K.; Oyabu, N.; Matsushige, K.; Yamada, H. Molecular-Scale Quantitative Charge Density Measurement of Biological Molecule by Frequency 
Modulation Atomic Force Microscopy in Aqueous Solutions. Nanotechnology 2015, 26, 285103.

(8) Ebeling, D.; Van den Ende, D.; Mugele, F. Electrostatic Interaction Forces in Aqueous Salt Solutions of Variable Concentration and Valency. Nanotechnology 2011, 22, 305706.

(9) Araki, Y.; Tsukamoto, K.; Takagi, R.; Miyashita, T.; Oyabu, N.; Kobayashi, K.; Yamada, H. Direct Observation of the Influence of Additives on Calcite Hydration by Frequency Modulation Atomic Force Microscopy. Cryst. Growth Des. 2014, 14, 62546260.

(10)Umeda, K.; Kobayashi, K.; Oyabu, N.; Matsushige, K.; Yamada, H. Molecular-Scale Quantitative Charge Density Measurement of Biological Molecule by Frequency Modulation Atomic Force Microscopy in Aqueous Solutions. Nanotechnology 2015, 26, 285103.

(11) Schlesinger, I.; Sivan, U. New Information on the Hydrophobic Interaction Revealed by Frequency Modulation AFM. Langmuir 2017, 33, 2485-2496.

(12) Vilhena, J. G.; Pimentel, C.; Pedraz, P.; Luo, F.; Serena, P. A.; Pina, C. M.; Gnecco, E.; Perez, R. Atomic-Scale Sliding Friction on Graphene in Water. ACS Nano 2016, 10, 4288-4293.

(13) Fukuma, T.; Higgins, M. J.; Jarvis, S. P. Direct Imaging of Individual Intrinsic Hydration Layers on Lipid Bilayers at Ångstrom Resolution. Biophys. J. 2007, 92, 3603-3609.

(14)De Beer, S.; Van den Ende, D.; Mugele, F. Dissipation and Oscillatory Solvation Forces in Confined Liquids Studied by Small-amplitude Atomic Force Spectroscopy. Nanotechnology 2010, 21, 325703 
(15)Herruzo, E. T.; Asakawa, H.; Fukuma, T.; Garcia, R. Three-dimensional Quantitative Force Maps in Liquid with 10 Piconewton, Angstrom and Sub-minute resolutions. Nanoscale 2013, 5, 2678-2685.

(16) Fukuma, T.; Ueda, Y.; Yoshioka, S.; Asakawa, H. Atomic-Scale Distribution of Water Molecules at the Mica-Water Interface Visualized by Three-Dimensional Scanning Force Microscopy. Phys. Rev. Lett. 2010, 104, 016101.

(17) Fukuma, T.; Reischl, B.; Kobayashi, N.; Spijker, P.; Canova, F. F.; Miyazawa, K., Foster, A. S. Mechanism of Atomic Force Microscopy Imaging of Three-Dimensional Hydration Structures at a Solid-Liquid Interface. Phys. Rev. B 2015, 92, 155412.

(18) Miyazawa, K.; Kobayashi, N.; Watkins, M.; Shluger, A. L.; Amano, K. I.; Fukuma, T. A Relationship between Three-Dimensional Surface Hydration Structures and Force Distribution Measured by Atomic Force Microscopy. Nanoscale 2016, 8, 7334-7342.

(19) Reischl, B.; Raiteri, P.; Gale, J. D.; Rohl, A. L. Can Point Defects in Surfaces in Solution be Atomically Resolved by Atomic Force Microscopy? Phys. Rev. Lett. 2016, 117, 226101. (20) Söngen, H.; Marutschke, C.; Spijker, P.; Holmgren, E.; Hermes, I.; Bechstein, R.; Klassen, S.; Tracey, J.; Foster, A.S.; Kühnle, A. Chemical Identification at the Solid-Liquid Interface. Langmuir 2016, 33, 125-129.

(21) Ito, F.; Kobayashi, K.; Spijker, P.; Zivanovic, L.; Umeda, K.; Nurmi, T.; Holmberg, N.; Laasonen, Kari; Foster, A. S. ; Yamada, H. Molecular Resolution of the Water Interface at an Alkali Halide with Terraces and Steps. J. Phys. Chem. C 2016, 120, 19714-19722.

(22) Miyazawa, K.; Watkins, M.; Shluger, A. L.; Fukuma, T. Influence of Ions on TwoDimensional and Three-Dimensional Atomic Force Microscopy at Fluorite-Water Interfaces. Nanotechnology 2017, 28, 245701. 
(23) Ricci, M.; Spijker, P.; Stellacci, F.; Molinari, J. F.; Voïtchovsky, K. Direct Visualization of Single Ions in the Stern Layer of Calcite. Langmuir 2013, 29, 2207-2216.

(24) Kuwahara, Y. Muscovite Surface Structure Imaged by Fluid Contact Mode AFM. Phys. Chem. Miner. 1999, 26, 198-205.

(25) Fukuma, T.; Kobayashi, K.; Matsushige, K.; Yamada, H. True Atomic Resolution in Liquid by Frequency-Modulation Atomic Force Microscopy. Appl. Phys. Lett. 2005, 87,034101 .

(26) Voïtchovsky, K. Anharmonicity, Solvation Forces, and Resolution in Atomic Force Microscopy at the Solid-Liquid Interface. Phys. Rev. E 2013, 88, 022407.

(27) Ebeling, D.; Solares, S. D. Amplitude Modulation Dynamic Force Microscopy Imaging in Liquids with Atomic Resolution: Comparison of Phase Contrasts in Single and Dual Mode Operation. Nanotechnology 2013, 24, 135702.

(28) Schlegel, M. L.; Nagy, K. L.; Fenter, P.; Cheng, L.; Sturchio, N. C.; Jacobsen, S. D. Cation Sorption on the Muscovite (001) Surface in Chloride Solutions Using High-Resolution XRay Reflectivity. Geochim. Cosmochim. Acta 2006, 70, 3549-3565.

(29) Park, C.; Fenter, P. A.; Sturchio, N. C.; Nagy, K. L. Thermodynamics, Interfacial Structure, and $\mathrm{pH}$ Hysteresis of $\mathrm{Rb}+$ and $\mathrm{Sr} 2+$ Adsorption at the Muscovite (001)- Solution Interface. Langmuir 2008, 24, 13993-14004.

(30) Lee, S. S.; Fenter, P.; Nagy, K. L.; Sturchio, N. C. Real-Time Observation of Cation Exchange Kinetics and Dynamics at the Muscovite-Water Interface. Nat. Commun. 2017, 8,15826

(31) Kobayashi, K.; Oyabu, N.; Kimura, K.; Ido, S.; Suzuki, K., Imai, T.; Tagami, K.; Tsukada, M.; Yamada, H. Visualization of Hydration Layers on Muscovite Mica in Aqueous 
Solution by Frequency-Modulation Atomic Force Microscopy. J. Chem. Phys. 2013, 138, 184704 .

(32) Kimura, K.; Ido, S.; Oyabu, N.; Kobayashi, K.; Hirata, Y.; Imai, T.; Yamada, H. Visualizing Water Molecule Distribution by Atomic Force Microscopy. J. Chem. Phys. 2010, 132, 194705.

(33) Asakawa, H.; Yoshioka, S.; Nishimura, K. I.; Fukuma, T. Spatial Distribution of Lipid Headgroups and Water Molecules at Membrane/Water Interfaces Visualized by ThreeDimensional Scanning Force Microscopy. ACS Nano 2012, 6, 9013-9020.

(34) Martin-Jimenez, D.; Chacon, E.; Tarazona, P.; Garcia, R. Atomically Resolved ThreeDimensional Structures of Electrolyte Aqueous Solutions near a Solid Surface. Nat. Commun. 2016, 7, 12164

(35) Garcia, R.; San Paulo, A. Attractive and Repulsive Tip-Sample Interaction Regimes in Tapping-Mode Atomic Force Microscopy. Phys. Rev. B 1999, 60, 4961.

(36) Martinez, N.F.; Garcia, R. Measuring Phase Shifts and Energy Dissipation with an Amplitude Modulation AFM, Nanotechnology 2006, 17, S167.

(37) Hölscher, H. Quantitative Measurement of Tip-Sample Interactions in Amplitude Modulation Atomic Force Microscopy. Appl. Phys. Lett. 2006, 89, 123109.

(38) Payam, A. F.; Martin-Jimenez, D.; Garcia, R. Force Reconstruction from Tapping Mode Force Microscopy Experiments. Nanotechnology 2015, 26, 185706.

(39) Argyris, D.; Ashby, P. D.; Striolo, A. Structure and Orientation of Interfacial Water Determine Atomic Force Microscopy Results: Insights from Molecular Dynamics Simulations. ACS Nano 2011, 5, 2215-2223. 
(40) Watkins, M.; Berkowitz, M. L.; Shluger, A. L. Role of Water in Atomic Resolution AFM in Solutions. Phys. Chem. Chem. Phys. 2011, 13, 12584-12594.

(41) Watkins, M; Reischl, B. A Simple Approximation for Forces Exerted on an AFM Tip in Liquid. J. Chem. Phys. 2013, 138, 154703. 